OPEN ACCESS

Edited by: Tzung-Cheng Huan,

National Chiayi University, Taiwan

Reviewed by:

Zacharoula Karabouta, University General Hospital of

Thessaloniki AHEPA, Greece Eleni Andreou,

University of Nicosia, Cyprus

*Correspondence:

Dimitrios Papandreou

Dimitrios.papandreou@zu.ac.ae

Specialty section:

This article was submitted to

Environmental Psychology, a section of the journal

Frontiers in Psychology

Received: 10 April 2020

Accepted: 23 April 2020

Published: 03 June 2020

Citation:

Saleh A, Alhadhrami JS, Al Ramahi MS, Albloushi HA, Hijazi R, Abboud M and Papandreou D (2020) Emirati Adults Have a Higher Overall Knowledge on Vitamin D Compared to Tourists. Front. Psychol. 11:1022. doi: 10.3389/fpsyg.2020.01022

\section{Emirati Adults Have a Higher Overall Knowledge on Vitamin D Compared to Tourists}

\author{
Ahlam Saleh ${ }^{1}$, Jawaher Saeed Alhadhrami', Maei Saeed Al Ramahi', \\ Halima Ali Albloushi', Rafiq Hijazi ${ }^{2}$, Myriam Abboud ${ }^{1}$ and Dimitrios Papandreou' ${ }^{1 *}$ \\ ${ }^{1}$ Department of Health Sciences, College of Natural and Health Sciences, Zayed University Abu Dhabi, Abu Dhabi, United \\ Arab Emirates, ${ }^{2}$ Department of Math, College of Natural and Health Sciences, Zayed University Abu Dhabi, Abu Dhabi, \\ United Arab Emirates
}

Objectives: In the last decade, vitamin D deficiency has become a major global issue. One of the main functions of vitamin $D$ is the proper absorption of calcium in the gastrointestinal track. Optimal vitamin $\mathrm{D}$ levels are mandatory for adequate calcium absorption and bone health. The purpose of this study was to assess the level of knowledge of vitamin D, calcium, and physical activity among Emirati and tourist adults in Abu Dhabi.

Methods: This is a cross-sectional study that took place in three different malls in Abu Dhabi and included Emirati and tourist adults. Participants were asked to fill in a questionnaire consisting of 32 questions. These included questions on vitamin $\mathrm{D}$, calcium, supplement, and physical activity knowledge. Another section of the questionnaire included general information on age, sex, education, weight, and height. The collected data were statistically analyzed using descriptive statistics using IBM SPSS statistics for Windows version 26.0 (SPSS Inc., Chicago, IL, United States). Statistical significance was set at $P<0.05$.

Results: Out of 147 adults, 113 were females and 34 males. The mean age, height, and weight were $27.9 \pm 8.6$ years, $162.7 \pm 10.4 \mathrm{~cm}$, and $66.5 \pm 19.5 \mathrm{~kg}$, respectively. Emiratis had statistically significant higher basic knowledge on vitamin D compared to tourists (44.9 vs $27.1 \%$ ), respectively. More than $66 \%$ of the whole sample was aware that vitamin $\mathrm{D}$ deficiency can affect muscle strength, as well as that calcium may affect osteoporosis. In a multiple regression model to analyze the possible effects of other factors to knowledge, it was found that only age (Beta: 0.045, $P<0.014$ ) and nationality (Beta: 0.750, $P<0.018$ ) were independently and significantly associated to vitamin D.

Conclusion: Emirati participants showed a higher overall vitamin D knowledge than their tourist counterparts. Both groups had low/medium level of knowledge when it comes to physical activity and calcium and vitamin D supplements.

Keywords: knowledge, vitamin D, calcium, physical activity, nutrition, Emiratis, tourists 


\section{INTRODUCTION}

Vitamin D and calcium levels have been a major concern in the last 20 years, and many studies have been conducted to analyze how diet and supplements may provide a beneficial effect especially to those people who are deficient (Holick and Chen, 2008; Alemu and Varnam, 2012). Vitamin D deficiency in both children and adults poses a major global health issue (Wahl et al., 2012).

Vitamin D mainly contributes to the regulation of calcium and phosphate metabolism and the maintenance of a healthy skeleton. Humans synthesize vitamin D primarily from sun exposure; however, other sources of vitamin $\mathrm{D}$ are provided through intake of selected foods and nutritional supplements (Charoenngam et al., 2019). Deficiency of vitamin D is very prevalent in the Middle East region, and it has recently reached $81 \%$ among various age groups (Mithal et al., 2009; Holick, 2017).

There are two forms of vitamin $\mathrm{D}$ : vitamin $\mathrm{D}_{3}$ and vitamin $\mathrm{D}_{2}$. Both precursors come from diet and sunlight and are finally converted in the kidney to form 1, 25-dihydroxyvitamin D (calcitriol), which is the active form (Zhang and Naughton, 2010). The Endocrine Society's Clinical Practice Guideline defines vitamin D deficiency, insufficiency, and sufficiency as serum concentrations of $25(\mathrm{OH}) \mathrm{D}$ of $<20,21-29$, and $30-$ $100 \mathrm{ng} / \mathrm{ml}$, respectively.

One of the major functions of vitamin $\mathrm{D}$ is the absorption of calcium in the gastrointestinal track. Optimal vitamin D levels are mandatory for adequate calcium absorption. It has been observed that the body can only absorb $10-15 \%$ if vitamin D status is not adequate (Khazai et al., 2008).

In addition, physical activity (PA) is an important factor in health promotion and disease prevention. Lack of PA has been shown to contribute to the development of many chronic diseases such as diabetes type 2 and cardiovascular disease. Consequently, PA guidelines have been developed to give people knowledge about what is, in general, a minimum amount of PA to improve health. The recommendations concluded that adults should exercise for $30 \mathrm{~m}$ on most days of the week (Vaara et al., 2019). The lack of knowledge on vitamin $\mathrm{D}$, calcium, and PA could be a potential risk factor for the development of non-communicable diseases such as osteoporosis, cardiovascular disease, and cancer. Data from United Kingdom, Saudi Arabia, United Arab Emirates, and Kuwait report mixed results on the lack of awareness and knowledge on vitamin D (Alemu and Varnam, 2012; Al-Mutairi et al., 2012; Salmanpour et al., 2016; Zhou et al., 2016; Alamoudi et al., 2019; Almuqati et al., 2019).

The purpose of this study was to assess the overall knowledge on vitamin D, calcium, supplements, and PA among Emirati and tourist adults in Abu Dhabi.

\section{MATERIALS AND METHODS}

This is a cross-sectional study that took place in three different malls in Abu Dhabi and included Emirati and tourist adults. Initially, it was scheduled to invite 200 adults to participate, but due to the coronavirus disease, we succeeded to collect only 147 subjects (98 Emiratis and 49 tourists).

The inclusion criteria were age $>18$ years old, not taking any medication or vitamin supplements, and not having a medical condition such as cardiovascular disease, diabetes, or osteoporosis. The researchers were waiting outside of malls and were randomly approaching the subjects. First some basic questions were asked to make sure the selected participants were qualified to participate in the study; then, they were administered with the questionnaire. The questionnaire included 32 basic knowledge questions on vitamin $\mathrm{D}$, calcium, vitamin $\mathrm{D}$ and calcium supplements, and PA knowledge. Another section of the questionnaire included general information about the characteristics of the participants such as age, sex, education, weight, and height.

The level of knowledge was defined as Low/Medium/High, where Low was $<40 \%$ correct responses to all questions, Medium from 41 to $70 \%$, and High $>70 \%$. The study was approved by the ethical committee of Zayed University, and all participants signed a consent form.

\section{Statistical Analysis}

The IBM SPSS Statistics (version 26) was used to enter, prepare, and analyze the collected data. Descriptive statistics including the means and standard deviations were reported for numerical data, and the frequencies and percentages were used to summarize the categorical data. The Chi-square test of homogeneity was used to test for differences in individual knowledge questions between Emiratis and tourists, while the independent twosample t-test was employed to examine differences in the knowledge components between Emiratis and tourists. Statistical significance was set at $P<0.05$.

\section{RESULTS}

Table 1 presents the characteristics of the sample. Out of 147 adults, 113 were females and 34 males. The mean age, height, and weight were $27.9 \pm 8.6$ years, $162.7 \pm 10.4 \mathrm{~cm}$, and $66.5 \pm 19.5 \mathrm{~kg}$, respectively. Out of the total sample, $67.3 \%$ were Emirati and $32.7 \%$ were tourists, while $39.1 \%$ were overweight and obese.

Table 2 compares the knowledge of Emiratis and tourists in all four categories. Emiratis had statistically significantly higher basic knowledge on vitamin D compared to tourists (44.9 vs 27.1\%). Both Emirati and tourists had low/medium knowledge on PA and supplements.

The individual's statistically significant responses on knowledge questions are presented in Table 3. More than $66 \%$ of the whole sample were aware that vitamin D deficiency can affect muscle strength, as well as that calcium may affect osteoporosis. Emirati had higher knowledge in six out of nine questions compared to tourist ones.

Table 4 shows the comparison of overall score of knowledge among Emirati and tourist sample. Emirati had statistically significantly higher knowledge on vitamin D compared to that of tourists. No significant differences were found on the other three parameters when comparing the two groups. 
TABLE 1 | Characteristics of study participants according to gender ( $n=147)$.

\begin{tabular}{|c|c|c|c|c|c|c|}
\hline & \multicolumn{2}{|c|}{ Male $(n=34)$} & \multicolumn{2}{|c|}{ Female $(n=113)$} & \multicolumn{2}{|c|}{ Total $(n=147)$} \\
\hline & Frequency & Percentage & Frequency & Percentage & Frequency & Percentage \\
\hline Age (years) & \multicolumn{2}{|c|}{$32.2 \pm 9.8$} & \multicolumn{2}{|c|}{$26.6 \pm 7.8$} & \multicolumn{2}{|c|}{$27.9 \pm 8.6$} \\
\hline Height (cm) & \multicolumn{2}{|c|}{$172.1 \pm 9.8$} & \multicolumn{2}{|c|}{$159.8 \pm 8.9$} & \multicolumn{2}{|c|}{$162.7 \pm 10.4$} \\
\hline Weight (KG) & \multicolumn{2}{|c|}{$80.6 \pm 24.2$} & \multicolumn{2}{|c|}{$62.2 \pm 15.7$} & \multicolumn{2}{|c|}{$66.5 \pm 19.5$} \\
\hline \multicolumn{7}{|l|}{ BMI } \\
\hline Underweight & 1 & 2.9 & 13 & 11.5 & 14 & 9.5 \\
\hline Normal & 14 & 41.2 & 60 & 53.1 & 74 & 50.3 \\
\hline Overweight & 11 & 32.4 & 20 & 17.7 & 31 & 21.1 \\
\hline Obese & 8 & 23.5 & 20 & 17.7 & 28 & 19.0 \\
\hline \multicolumn{7}{|l|}{ Nationality } \\
\hline Emirati & 14 & 41.2 & 85 & 75.2 & 99 & 67.3 \\
\hline Tourists & 20 & 58.8 & 28 & 24.8 & 48 & 32.7 \\
\hline \multicolumn{7}{|l|}{ Education level } \\
\hline High School or less & 9 & 26.5 & 27 & 23.9 & 36 & 24.5 \\
\hline Diploma & 3 & 8.8 & 20 & 17.7 & 23 & 15.6 \\
\hline Bachelor & 15 & 44.1 & 55 & 48.7 & 70 & 47.6 \\
\hline Postgraduate & 7 & 20.6 & 11 & 9.7 & 18 & 12.2 \\
\hline \multicolumn{7}{|l|}{ Taking medication } \\
\hline Yes & 6 & 17.6 & 18 & 15.9 & 24 & 16.3 \\
\hline No & 28 & 82.4 & 95 & 84.1 & 123 & 83.7 \\
\hline
\end{tabular}

Data expressed as mean \pm standard deviation.

TABLE 2 | Comparison of knowledge level between Emirati and tourists.

\begin{tabular}{|c|c|c|c|c|}
\hline \multirow[t]{2}{*}{ Knowledge component } & \multirow[t]{2}{*}{ Nationality } & \multicolumn{3}{|c|}{ Knowledge level } \\
\hline & & Low (\%) & Medium (\%) & High (\%) \\
\hline \multirow[t]{3}{*}{ Vitamin D knowledge } & Emirati & 4.1 & 51.0 & 44.9 \\
\hline & Tourists & 8.3 & 64.6 & 27.1 \\
\hline & Total & 5.5 & 55.5 & 39.0 \\
\hline \multirow[t]{3}{*}{ Calcium knowledge } & Emirati & 6.1 & 60.6 & 33.3 \\
\hline & Tourists & 12.5 & 62.5 & 25.0 \\
\hline & Total & 8.2 & 61.2 & 30.6 \\
\hline \multirow[t]{3}{*}{ Physical activity knowledge } & Emirati & 30.3 & 59.6 & 10.1 \\
\hline & Tourists & 25.0 & 47.9 & 27.1 \\
\hline & Total & 28.6 & 55.8 & 15.6 \\
\hline \multirow[t]{3}{*}{ Vitamin D and calcium supplements knowledge } & Emirati & 41.4 & 53.5 & 5.1 \\
\hline & Tourists & 43.8 & 52.1 & 4.2 \\
\hline & Total & 42.2 & 53.1 & 4.8 \\
\hline
\end{tabular}

Low (<40\%), Medium (41-70\%), High (>70\%).

Using a multiple regression model to analyze the possible effects of other factors to knowledge, it was found that only age (Beta: 0.045, $P<0.014$ ) and nationality (Beta: 0.750, $P<0.018)$ were independently and significantly associated to vitamin D (Table 5).

\section{DISCUSSION}

The current study shows that Emiratis have a higher overall knowledge on vitamin D compared to that of tourists.
Both groups showed low/medium knowledge on calcium, supplements, and PA. Many local and international countries have reported mixed results. The countries that showed low awareness on vitamin D may also explain why these countries have a high percentage of vitamin $\mathrm{D}$ deficient population. On the other hand, similar data from the same countries showed that their population had very high awareness on vitamin $\mathrm{D}$ levels, and their results are in agreement with our data too (Alemu and Varnam, 2012; Zhou et al., 2016; Almuqati et al., 2019).

In a United Kingdom study, almost $54 \%$ of the participants had low knowledge on the basic symptoms of vitamin D 
TABLE 3 | Statistically significant responses of the sample to individual questions.

\begin{tabular}{|c|c|c|c|}
\hline & Tourists (\%) & Emirati (\%) & $P$-value \\
\hline Q1: Can pancreatitis effect vitamin D absorption? & 45.8 & 67.7 & $0.011^{*}$ \\
\hline Q2: Do you think that the deficiency of vitamin D can lead to baldness? & 58.3 & 28.6 & $0.001^{* \star}$ \\
\hline Q3: Do you think that the deficiency of vitamin D can decrease muscle strength? & 79.2 & 91.9 & $0.027^{*}$ \\
\hline Q4: Do you think that vitamin D deficiency can lower bone density? & 50.0 & 77.8 & $0.001^{\star \star}$ \\
\hline Q5: Does alcohol have an effect on vitamin D absorption? & 68.8 & 85.9 & $0.015^{*}$ \\
\hline Q6: Do you think that animal sources (meats/egg) have more vitamin D than plant sources? & 50.0 & 69.7 & $0.02^{*}$ \\
\hline Q7: Does dietary calcium affect osteoporosis? & 81.3 & 96.0 & $0.003^{\star *}$ \\
\hline Q8: Are there any harmful effects if one takes more than $2,000 \mathrm{mg}$ of calcium? & 41.7 & 15.2 & $0.001^{\star *}$ \\
\hline Q9: Are vitamin D supplements safe, if you are not vitamin D deficient? & 68.8 & 51.5 & $0.048^{*}$ \\
\hline
\end{tabular}

*Statistically significant at $P<0.05$. ${ }^{* *}$ Statistically significant at $P<0.01$.

TABLE 4 | Comparison of overall score of knowledge among Emirati and tourist sample.

\begin{tabular}{|c|c|c|c|c|c|c|}
\hline Knowledge component & Nationality & $n$ & Mean & Standard deviation & $t$ & $P$-value \\
\hline \multirow[t]{2}{*}{ Vitamin D knowledge } & Emirati & 98 & 6.92 & 1.71 & 2.74 & $0.007^{*}$ \\
\hline & Expatriate & 48 & 6.06 & 1.88 & & \\
\hline \multirow[t]{2}{*}{ Calcium knowledge } & Emirati & 99 & 6.23 & 1.33 & 1.89 & 0.060 \\
\hline & Expatriate & 48 & 5.76 & 1.56 & & \\
\hline \multirow[t]{2}{*}{ Physical activity knowledge } & Emirati & 99 & 5.32 & 1.87 & -1.36 & 0.176 \\
\hline & Expatriate & 48 & 5.80 & 2.25 & & \\
\hline \multirow[t]{2}{*}{ Vitamin D and calcium supplements knowledge } & Emirati & 99 & 4.51 & 1.89 & -0.01 & 0.995 \\
\hline & Expatriate & 48 & 4.51 & 1.68 & & \\
\hline
\end{tabular}

*Statistically significant set at $P<0.05$.

TABLE 5 | Regression analysis for factors affecting knowledge levels.

\begin{tabular}{|c|c|c|c|c|c|c|c|c|c|c|c|c|}
\hline \multirow[t]{2}{*}{ Dependent variable } & \multicolumn{3}{|c|}{ Vitamin D knowledge } & \multicolumn{3}{|c|}{ Calcium knowledge } & \multicolumn{3}{|c|}{ Physical knowledge } & \multicolumn{3}{|c|}{ Supplements knowledge } \\
\hline & B & SE & $P$-value & B & SE & $P$-value & B & SE & $P$-value & B & SE & $P$-value \\
\hline Intercept & 3.297 & 0.824 & 0.000 & 5.770 & 0.843 & 0.000 & 3.797 & 0.653 & 0.000 & 2.832 & 0.585 & 0.000 \\
\hline Age & 0.045 & 0.018 & $0.014^{*}$ & 0.021 & 0.018 & 0.245 & -0.004 & 0.014 & 0.779 & 0.009 & 0.013 & 0.502 \\
\hline $\mathrm{BMl}$ & 0.024 & 0.022 & 0.281 & -0.003 & 0.023 & 0.894 & -0.003 & 0.018 & 0.855 & -0.022 & 0.016 & 0.161 \\
\hline Gender & 0.494 & 0.329 & 0.135 & 0.174 & 0.336 & 0.605 & -0.281 & 0.261 & 0.282 & -0.071 & 0.233 & 0.760 \\
\hline Taking medication & 0.321 & 0.356 & 0.369 & -0.087 & 0.364 & 0.811 & -0.042 & 0.282 & 0.882 & -0.154 & 0.253 & 0.544 \\
\hline Nationality & 0.750 & 0.312 & $0.018^{*}$ & 0.523 & 0.319 & 0.104 & -0.222 & 0.247 & 0.371 & 0.192 & 0.221 & 0.387 \\
\hline Diploma & -0.282 & 0.416 & 0.499 & 0.113 & 0.426 & 0.792 & 0.379 & 0.330 & 0.253 & 0.379 & 0.296 & 0.202 \\
\hline Bachelor & 0.189 & 0.320 & 0.556 & -0.059 & 0.327 & 0.857 & -0.066 & 0.253 & 0.794 & -0.004 & 0.227 & 0.985 \\
\hline Postgraduate & -1.198 & 0.484 & 0.014 & -0.583 & 0.495 & 0.241 & 0.205 & 0.384 & 0.593 & 0.697 & 0.343 & 0.044 \\
\hline$F$ & 3.180 & & & 0.802 & & & 0.664 & & & 1.137 & & \\
\hline$P$-value & 0.002 & & & 0.602 & & & 0.723 & & & 0.342 & & \\
\hline$R^{2}$ & $24.2 \%$ & & & $9.2 \%$ & & & $9.6 \%$ & & & $9.4 \%$ & & \\
\hline
\end{tabular}

*Statistical significance set at $P<0.05$.

deficiency, while $34 \%$ did not get enough sunlight, and $11 \%$ did not have enough vitamin D from food sources (Alemu and Varnam, 2012). A Chinese study assessing the knowledge of 515 students about vitamin D concluded that the participants had low knowledge levels on vitamin $\mathrm{D}$, and they should include vitamin-rich food sources in their diet (Zhou et al., 2016).

In a recent cross-sectional study in Saudi Arabia, 1,022 participants' knowledge on vitamin $\mathrm{D}$ was evaluated and it was concluded that there is a very high level of inadequate knowledge of vitamin $\mathrm{D}$ that is also related to the educational levels of the population (Alamoudi et al., 2019).

More recently, a cross-sectional study was also conducted in Sharjah, United Arab Emirates. A total of 503 adults were selected from public places using a convenience sampling method. Vitamin D deficiency knowledge was very common in the majority of the adults (Salmanpour et al., 2016). 
On the other hand, and in the neighboring country of Kuwait, investigations of the level of awareness, knowledge, and attitude of representative groups from the general population toward sun protection showed that the majority of the population had good knowledge toward sun protection and its relation to vitamin $\mathrm{D}$; however, low knowledge on calcium was observed (Al-Mutairi et al., 2012).

Similar results were also observed in another cross-sectional study that included students from Saudi Arabia. The authors evaluated 501 medical students and found that most students were aware of the risks of unprotected sun exposure. Almost 71\% of the students were familiar with the association of vitamin D level and sun exposure (Almuqati et al., 2019).

It is worthy to note that our study has many limitations: (a) It does not represent the population of Abu Dhabi, (b) the sample size was small, and (c) we did not have information whether the sample consumed any vitamin D or calcium supplements the last year. The last one could affect significantly the knowledge level. Nevertheless, our study provides some initial data about the knowledge level on vitamin $\mathrm{D}$ and the need for larger sample studies in the near future.

\section{CONCLUSION}

The study highlights a high knowledge level among the Emirati participants on dietary vitamin D compared to tourists; however,

\section{REFERENCES}

Alamoudi, L. H., Almuteeri, R. Z., Al-Otaibi, M. E., Alshaer, D. A., Fatani, S. K., Alghamdi, M. M., et al. (2019). Awareness of vitamin D deficiency among the general population in jeddah. saudi Arabia. J. Nutr. Metab. 2019:4138187. doi: $10.1155 / 2019 / 4138187$

Alemu, E., and Varnam, R. (2012). Awareness of vitamin D deficiency among at-risk patients. BMC Res. Notes 5:17. doi: 10.1186/1756-050 0-5-17

Almuqati, R. R., Alamri, A. S., and Almuqati, N. R. (2019). Knowledge, attitude, and practices toward sun exposure and use of sun protection among nonmedical, female, university students in Saudi Arabia: a cross-sectional study. Int. J. Womens Dermatol. 5, 105-109. doi: 10.1016/j.ijwd.2018.11.005

Al-Mutairi, N., Issa, B. I., and Nair, V. (2012). Photoprotection and vitamin D status: a study on awareness, knowledge and attitude towards sun protection in general population from Kuwait, and its relation with vitamin D levels. Indian J. Dermatol. Venereol. Leprol. 78, 342-349. doi: 10.4103/0378-6323. 95451

Charoenngam, N., Shirvani, A., and Holick, M. F. (2019). Vitamin D for skeletal and non-skeletal health: what we should know. J. Clin. Orthop. Trauma 10, 1082-1093. doi: 10.1016/j.jcot.2019.07.004

Holick, M. F. (2017). The vitamin D deficiency pandemic: approaches for diagnosis, treatment and prevention. Rev. Endocr. Metab. Disord. 18, 153-165. doi: 10. 1007/s11154-017-9424-1

Holick, M. F., and Chen, T. C. (2008). Vitamin D deficiency: a worldwide problem with health consequences. Am. J. Clin. Nutr. 87, 1080S-1086S. doi: 10.1093/ ajcn/87.4.1080S

Khazai, N., Judd, S. E., and Tangpricha, V. (2008). Calcium and vitamin D: skeletal and extraskeletal health. Curr. Rheumatol. Rep. 10, 110-117. doi: 10.1007/ s11926-008-0020-y a low/medium knowledge level of both groups on calcium, vitamin $\mathrm{D}$ and calcium supplements, and PA was observed. Future intervention studies on a large sample are needed to accurately investigate the level of awareness in order to determine the possible reasons for the low knowledge of calcium and PA.

\section{DATA AVAILABILITY STATEMENT}

The data used to support the findings of this study are available upon request from the corresponding author.

\section{ETHICS STATEMENT}

The studies involving human participants were reviewed and approved by the Research Ethical Committee, Zayed University. The patients/participants provided their written informed consent to participate in this study.

\section{AUTHOR CONTRIBUTIONS}

$\mathrm{RH}$ and MA contributed to the conception, design, and statistical analysis of the study. AS, JA, MA, and HA collected the data and organized the database. DP contributed to the conception and design of the study and wrote the first draft of the manuscript. All authors contributed to manuscript revision and approval.

Mithal, A., Wahl, D. A., Bonjour, J. P., Burckhardt, P., Dawson-Hughes, B., Eisman, J. A., et al. (2009). Global vitamin D status and determinants of hypovitaminosis D. Osteoporos Int. 20, 1807-1820. doi: 10.1007/s00198-009-0954-6

Salmanpour, V. A., Ibrahim, H. S., Salameh, A. G., Yahya, A. M., and Debal, B. K. (2016). Vitamin D deficiency: knowledge and practices among the adult population in Sharjah. United Arab Emirates. Arch. Osteoporos 11:15. doi: 10. 1007/s11657-016-0269-0

Vaara, J. P., Vasankari, T., Koski, H. J., and Kyröläinen, H. (2019). Awareness and knowledge of physical activity recommendations in young adult Men. Front. Public Health. 7:310. doi: 10.3389/fpubh.2019.00310

Wahl, D. A., Cooper, C., Ebeling, P. R., Eggersdorfer, M., Hilger, J., Hoffmann, K., et al. (2012). A global representation of vitamin D status in healthy populations. Arch. Osteoporos 7, 155-172. doi: 10.1007/s11657-012-0093-0

Zhang, R., and Naughton, D. P. (2010). Vitamin D in health and disease: current perspectives. Nutr. J. 9:65. doi: 10.1186/1475-2891-9-65

Zhou, M., Zhuang, W., Yuan, Y., Li, Z., and Cai, Y. (2016). Investigation on vitamin $\mathrm{D}$ knowledge, attitude and practice of university students in Nanjing. China. Public Health Nutr. 19, 78-82. doi: 10.1017/S1368980015000373

Conflict of Interest: The authors declare that the research was conducted in the absence of any commercial or financial relationships that could be construed as a potential conflict of interest.

Copyright (C) 2020 Saleh, Alhadhrami, Al Ramahi, Albloushi, Hijazi, Abboud and Papandreou. This is an open-access article distributed under the terms of the Creative Commons Attribution License (CC BY). The use, distribution or reproduction in other forums is permitted, provided the original author(s) and the copyright owner(s) are credited and that the original publication in this journal is cited, in accordance with accepted academic practice. No use, distribution or reproduction is permitted which does not comply with these terms. 